\title{
Relações entre cátions trocáveis do solo e suas correlações com a quali- dade de frutos de melão
}

\author{
Neyton de Oliveira Miranda; José F. de Medeiros; Sérgio Luiz A. Levien \\ UFERSA-Depto. Ciências Ambientais, Av. Francisco Mota, s/n, 59625-900 Mossoró-RN; neyton@ufersa.edu.br
}

\begin{abstract}
RESUMO
A qualidade dos frutos de melão pode ser prejudicada pelo excesso de nutrientes no solo e pelo desequilíbrio entre eles, causados por adubações excessivas e desuniformes. A amostragem em duas áreas de produção de melão foi realizada para identificar entre relações catiônicas do solo (K:(Ca+Mg), K:Ca, K:Mg, K:CTC, Ca:Mg, Ca:CTC, Mg:CTC, Na:Ca, Na:Mg e PST) aquelas melhor correlacionadas com características de qualidade (espessura de polpa, firmeza da polpa e teor de sólidos solúveis totais). Para tanto se utilizou a estatística descritiva, o coeficiente de correlação de Spearman e a regressão múltipla. As variáveis de qualidade de frutos de melão apresentaram poucas correlações com as relações catiônicas do solo. No Goldex, as melhores correlações foram de $\mathrm{K}$ :CTC com espessura de polpa; Ca:CTC e Mg:CTC com firmeza de polpa e K:CTC, K:Ca, K:(Ca+Mg), Na:Ca e Na:Mg com SST, todas positivas. No Orange Flesh, observaram-se correlações apenas de firmeza de polpa com Ca:CTC (positiva), $\mathrm{K}: \mathrm{Mg}, \mathrm{K}: \mathrm{Ca}$, $\mathrm{K}:(\mathrm{Ca}+\mathrm{Mg})$ e Na:Ca (negativas). No Orange Flesh, através da contribuição para as regressões, identificou-se como mais importantes as relações catiônicas Ca:CTC para espessura $(6,2 \%)$ e firmeza de polpa (10,9\%), e Mg:CTC para o teor de sólidos solúveis totais $(1,5 \%)$
\end{abstract}

Palavras-chave: Cucumis melo, firmeza de polpa, sólidos solúveis totais.

\begin{abstract}
Soil cationic ratios and its correlation with melon fruit quality

Melon fruit quality can be reduced by soil nutrient excess and imbalance, both caused by excessive and non uniform fertilizations. Soil samples were taken from two melon fields aiming to identify, among soil cationic ratios $(\mathrm{K}:(\mathrm{Ca}+\mathrm{Mg}), \mathrm{K}: \mathrm{Ca}, \mathrm{K}: \mathrm{Mg}, \mathrm{K}: \mathrm{CTC}$, Ca:Mg, Ca:CTC, Mg:CTC, Na:Ca, Na:Mg, and ESP), those better correlated with fruit quality characteristics (pulp thickness, pulp firmness and total soluble solids (SST)). Descriptive statistics, Spearman's correlation and multiple regressions were used in the analysis. Melon fruit quality characteristics presented few correlation with soil cationic ratios. In Goldex, the best correlation found were of K:CTC with pulp fruit thickness; $\mathrm{Ca}$ :CTC and Mg:CTC with pulp firmness and of $\mathrm{K}: \mathrm{CTC}, \mathrm{K}: \mathrm{Ca}, \mathrm{K}:(\mathrm{Ca}+\mathrm{Mg}), \mathrm{Na}: \mathrm{Ca}$ and $\mathrm{Na}: \mathrm{Mg}$ with SST, all positive. In Orange Flesh, only pulp firmness showed correlation with $\mathrm{Ca}: \mathrm{CTC}$ (positive), $\mathrm{K}: \mathrm{Mg}, \mathrm{K}: \mathrm{Ca}, \mathrm{K}:(\mathrm{Ca}+\mathrm{Mg}$ ) and $\mathrm{Na}: \mathrm{Ca}$ (negative). On the basis of their contribution to regressions in Orange Flesh, cationic ratios identified as more important were Ca:CTC for pulp fruit thickness $(6,2 \%)$ and pulp firmness $(10,9 \%)$, and $\mathrm{Mg}: \mathrm{CTC}$ for total soluble solids (1,5\%).
\end{abstract}

Keywords: Cucumis melo, pulp firmness, total soluble solids

\section{(Recebido para publicação em 6 de agosto de 2007; aceito em 23 de abril de 2008)}

$\mathrm{O}$ desempenho do melão é importante para o agronegócio brasileiro tanto no mercado interno como internacional (Crisóstomo et al., 2002). Os estados do Rio Grande do Norte, Ceará, Bahia e Pernambuco produzem mais de $90 \%$ do melão brasileiro, destacandose as regiões de Mossoró, Assú e Baraúna, no Rio Grande do Norte, e Baixo Jaguaribe no Ceará. Nestas regiões, a qualidade dos frutos produzidos atende aos requisitos básicos do mercado internacional, porém está abaixo da média dos países concorrentes, dificultando a obtenção de melhores preços (Silva \& Costa, 2003).

A qualidade dos frutos de melão é prejudicada pelo desequilíbrio entre nutrientes no solo e por adubações excessivas e desuniformes, as quais, além de acumular no solo os nutrientes não assimilados pelas plantas nem lixiviados, originam regiões no campo com fertilidade diferente da média utilizada para recomendação de corretivos e fertilizantes. Por isto, mesmo em campos com fertilidade média adequada, a qualidade dos produtos agrícolas pode ser reduzida em regiões com $\mathrm{pH}$ e teores de nutrientes inadequados (Silva, 2000; França et al., 2000). Tal fato foi confirmado por Miranda et al. (2005), que associaram a variabilidade espacial da qualidade dos frutos de melão com características químicas e físicas dos solos, fossem estas inerentes ao solo, ou causadas por práticas inadequadas de manejo, como a aplicação desuniforme de água, fertilizantes e corretivos (Gonçalves et al., 1999; Schlindwein \& Anghinoni, 2000; Bergez \& Nolleau, 2003).

Em cultivos do meloeiro em alguns solos de origem calcária do $\mathrm{RN}$ e $\mathrm{CE}$, irrigados com água rica em $\mathrm{Ca}^{2+} \mathrm{e}$ com aplicações elevadas de $\mathrm{K}_{2} \mathrm{O}$, observa-se deficiência de $\mathrm{K}^{+}$para a cultura, devido à inibição competitiva da absorção de $\mathrm{K}^{+}$pelos altos teores de $\mathrm{Ca}^{2+}$ no solo (Crisóstomo et al., 2002). Por outro lado, altas concentrações de $\mathrm{Ca}^{2+}$ e $\mathrm{K}^{+}$ podem inibir a absorção de $\mathrm{Mg}^{2+}$, diminuir sua translocação da raiz à parte aérea, e, assim, causar sua deficiência. Isto

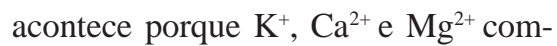
petem pelos mesmos sítios de absorção na raiz, de maneira que o cátion em maior concentração na solução do solo tem absorção preferencial em detrimento dos outros. Além disto, teores elevados de cátions monovalentes na solução do solo podem induzir deficiência dos divalentes, que são retidos mais fortemente pelo complexo de troca do solo (Malavolta et al., 1997).

O conceito de relações catiônicas ideais surgiu, segundo Kelling \& Peters 
(2004), da observação de que solos com baixos teores de $\mathrm{K}^{+}$trocável não respondiam à adubação potássica, enquanto outros com teores satisfatórios respondiam. Segundo os autores, testes com várias relações não comprovaram este conceito, nem determinaram o benefício econômico de adubações feitas para atingir tal relação. $\mathrm{O}$ enfoque do nível de suficiência, que recomenda teores suficientes, mas não excessivos de cada cátion em vez de tentar obter uma relação favorável de saturação dos cátions, é suportado por maior base de pesquisa e tem apresentado melhores resultados agronômicos e econômicos. Neste sentido, os autores indicam faixas de saturação de cada cátion, as quais pouco afetariam o crescimento e produtividade das culturas: 65 a $85 \%$ de $\mathrm{Ca}^{2+}, 6$ a $12 \%$ de $\mathrm{Mg}^{2+}$ e 2 a $5 \%$ de $\mathrm{K}^{+}$, com o $\mathrm{H}^{+}$ocupando os sítios restantes.

Este trabalho foi desenvolvido com o objetivo de identificar as relações catiônicas do solo que melhor correlacionam com atributos indicadores de qualidade, para identificar aquelas que possam estar influenciando a qualidade dos frutos de melão.

\section{MATERIAL E MÉTODOS}

Os dados foram coletados no ano de 2002 em duas áreas de produção comercial de melão em Mossoró-RN (05 $09^{\circ}$ 59,55" latitude sul, 37 $24^{\circ}$ '39,92" longitude oeste), nas quais o híbrido Goldex foi cultivado em Latossolo VermelhoAmarelo Eutrófico (LVA) e o híbrido Orange Flesh em Argissolo VermelhoAmarelo (PVA). O clima de Mossoró é do tipo BSwh' de Köppen (seco, muito quente com a estação chuvosa no verão, atrasando-se para o outono), com precipitação média anual de 500 a $600 \mathrm{~mm}$.

As características químicas e físicas dos solos LVA e PVA foram, à época de realização das amostragens, respectivamente: $\mathrm{pH}=6,81$ e 7,00; matéria orgânica $=16,52$ e $13,32 \mathrm{~g} \mathrm{dm}^{-3} ; \mathrm{P}=126,08$ e $55,68 \mathrm{mg} \mathrm{dm}^{-3} ; \mathrm{K}^{+}=23,68$ e 4,81 $\mathrm{mmol} \mathrm{dm}{ }^{-3} ; \mathrm{Ca}^{2+}=87,08$ e $65,33 \mathrm{mmol}$ $\mathrm{dm}^{-3} ; \mathrm{Mg}^{2+}=28,83$ e $12,87 \mathrm{mmol} \mathrm{dm}^{-3}$; $\mathrm{Na}^{+}=12,19$ e $6,97 \mathrm{mmol} \mathrm{dm}{ }^{-3}$; CTC $=$ 161,54 e $100,49 \mathrm{mmol} \mathrm{dm}^{-3}$; areia $=$ 577,54 e $872,27 \mathrm{~g} \mathrm{~kg}^{-1}$; silte $=98,62 \mathrm{e}$ $64,04 \mathrm{~g} \mathrm{~kg}^{-1}$; argila $=314,13$ e $63,83 \mathrm{~g}$ $\mathrm{kg}^{-1}$ e densidade do solo $=1,51$ e 1,62 $\mathrm{Mg} \mathrm{m}^{-3}$.

As mudas, produzidas em bandejas de poliestireno, foram transplantadas em espaçamento de 2,0 m entre linhas. No Goldex, a cada gotejador espaçado de $50 \mathrm{~cm}$ foram dispostas duas plantas a $10 \mathrm{~cm}$ uma da outra, porém a cada três gotejadores, um recebia apenas uma planta, totalizando 16.667 plantas por hectare; no Orange Flesh, a cada gotejador espaçado de $80 \mathrm{~cm}$ foram dispostas três plantas a $10 \mathrm{~cm}$ uma da outra, totalizando 18.750 plantas por hectare. Os tratos culturais seguiram o sistema de produção empregado na região.

A irrigação, manejada com base na evapotranspiração de referência calculada pelo método de Penman Monteith, utilizou tubos com gotejadores espaçados de $50 \mathrm{~cm}$ e vazão de $2,27 \mathrm{~L} \mathrm{~h}^{-1}$ no LVA, e espaçados de $80 \mathrm{~cm}$ com vazão de 5,0 L h'-1 no PVA. A lâmina bruta de irrigação aplicada foi de $322,8 \mathrm{~mm}$ no LVA e 316,7 mm no PVA. Foram fornecidos, através da fertirrigação, $69,8 \mathrm{~kg}$ $\mathrm{ha}^{-1}$ de $\mathrm{N}, 135,5 \mathrm{~kg} \mathrm{ha}^{-1}$ de $\mathrm{P}_{2} \mathrm{O}_{5}, 266,2$ $\mathrm{kg} \mathrm{ha}^{-1}$ de $\mathrm{K}_{2} \mathrm{O}, 1,4 \mathrm{~kg} \mathrm{ha}^{-1}$ de B, 1,3 kg $\mathrm{ha}^{-1}$ de $\mathrm{Mg}$ e 9,4 $\mathrm{kg} \mathrm{ha}^{-1}$ de S, no LVA. No PVA foram fornecidos $85,5 \mathrm{~kg} \mathrm{ha}^{-1}$ de $\mathrm{N}, 115,2 \mathrm{~kg} \mathrm{ha}^{-1}$ de $\mathrm{P}_{2} \mathrm{O}_{5}, 315,8 \mathrm{~kg}$ $\mathrm{ha}^{-1}$ de $\mathrm{K}_{2} \mathrm{O}, 4,8 \mathrm{~kg} \mathrm{ha}^{-1}$ de B, 5,8 $\mathrm{kg} \mathrm{ha}^{-1}$ de $\mathrm{Mg}$ e 4,7 $\mathrm{kg} \mathrm{ha}^{-1}$ de $\mathrm{S}$.

O solo de cada área foi amostrado na fase de pleno florescimento, na camada de 0 a $20 \mathrm{~cm}$, segundo uma malha com espaçamento regular de $20 \mathrm{~m}$ entre amostras, totalizando 75 parcelas no LVA e 56 parcelas no PVA. Após a colheita, os melões foram classificados em tipo exportação (PEXP), tipo mercado nacional (PNAC) e refugo. Foram determinadas a espessura da polpa (ESPOLP) com paquímetro; a firmeza de polpa, usando penetrômetro com "plunger" de $8 \mathrm{~mm}$ de diâmetro e o teor de sólidos solúveis totais (SST), utilizando refratômetro.

Os teores de $\mathrm{Ca}^{2+}, \mathrm{Mg}^{2+}, \mathrm{K}^{+}, \mathrm{Na}^{+}$e a acidez potencial $\left(\mathrm{H}^{+}+\mathrm{Al}^{3+}\right)$ das amostras de solo foram determinados segundo metodologias relatadas em Embrapa (1997). Calcularam-se as relações catiônicas: capacidade de troca de cátions (CTC), K:Ca, K:Mg, K:CTC, $\mathrm{K}:(\mathrm{Ca}+\mathrm{Mg}), \mathrm{Ca}: \mathrm{Mg}, \mathrm{Ca}: \mathrm{CTC}, \mathrm{Mg}: \mathrm{CTC}$, $\mathrm{Na}: \mathrm{Ca}, \mathrm{Na}: \mathrm{Mg}$ e percentagem de sódio trocável (PST), calculada pela equação PST $=\left(\mathrm{Na}^{+} /\right.$CTC $) \times 100$.

Os dados foram analisados através da estatística descritiva para verificar sua amplitude e variabilidade; teste de Shapiro-Wilk para verificar a normalidade; coeficiente de correlação de Spearman para identificar possível influência das relações catiônicas sobre as características de qualidade. Adotou-se o modelo de regressão linear múltipla sem o intercepto para avaliar a importância das relações catiônicas estudadas (K:Ca, K:Mg, K:(Ca+Mg), K:CTC, $\mathrm{Ca}: \mathrm{Mg}, \mathrm{Ca}$ CTC, Mg:CTC, $\mathrm{Na} \mathrm{Ca}$, $\mathrm{Na}: \mathrm{Mg}$ e PST) em explicar as características de qualidade dos frutos de melão (espessura de polpa (ESPOLP), firmeza da polpa e teor de sólidos solúveis totais (SST)). O modelo de melhor ajuste foi selecionado pelo procedimento passo a passo com teste para saída de variáveis (stepwise backward), no qual o modelo inicial contém todas as variáveis independentes e, a cada passo, a variável de menor importância é eliminada, até restarem no modelo apenas variáveis significativas. Calculou-se a soma dos quadrados do tipo II, na qual o efeito de uma variável do modelo é ajustado para todos os outros efeitos. $\mathrm{O}$ coeficiente de determinação parcial (\%) foi calculado para indicar quanto da variação total é explicado pelo efeito de uma variável ajustada para as demais.

\section{RESULTADOS E DISCUSSÃO}

A Tabela 1 apresenta os valores médios das características de qualidade, os quais, para espessura de polpa, foram 4,05 cm no Goldex e 3,90 cm no Orange Flesh. De acordo com os padrões citados por Filgueiras et al. (2000), a firmeza de polpa $(53,12 \mathrm{~N}$ do Orange Flesh e 40,21 N do Goldex) foi elevada; o conteúdo de sólidos solúveis totais do Goldex $(12,19 \%)$ foi adequado, mas o Orange Flesh $(9,10 \%)$ apresentou alguns valores baixos. Apesar da baixa variabilidade destas características, a ocorrência de regiões no campo com produtos de baixa qualidade pode causar redução considerável de receita (Upadhyaya et al., 1999).

Os parâmetros de estatística descritiva da Tabela 1, confrontados com os 
Tabela 1. Estatística descritiva de características de qualidade dos frutos de melão Goldex e Orange Flesh e de algumas relações catiônicas dos solos amostrados (descriptive statistics of quality characterisitcs of Goldex and Orange Flesh melons and of some soil cationic ratios). Mossoró, UFERSA, 2002.

\begin{tabular}{|c|c|c|c|c|c|c|c|c|c|c|}
\hline \multirow{2}{*}{ Variável } & \multicolumn{5}{|c|}{ Híbrido Goldex - Latossolo } & \multicolumn{5}{|c|}{ Híbrido Orange Flesh - Argissolo } \\
\hline & Média & Máx. & Mín. & CV & $\mathbf{W}$ & Média & Máx. & Mín. & CV & $\mathbf{W}$ \\
\hline Espessura (cm) & 4,05 & 4,62 & 3,60 & 5,10 & $0,98^{\text {ns }}$ & 3,90 & 4,95 & 3,12 & 8,13 & $0,98^{\text {ns }}$ \\
\hline Firmeza (N) & 40,21 & 47,68 & 31,67 & 8,17 & $0,98^{\mathrm{ns}}$ & 53,12 & 59,61 & 47,42 & 5,57 & $0,97^{\text {ns }}$ \\
\hline SST (\%) & 12,19 & 14,65 & 9,95 & 7,73 & $0,99^{\text {ns }}$ & 9,10 & 10,57 & 7,52 & 8,51 & $0,96^{\text {ns }}$ \\
\hline $\mathrm{K}: \mathrm{Ca}$ & 0,27 & 0,41 & 0,17 & 23,45 & $0,94^{* *}$ & 0,08 & 0,14 & 0,03 & 35,46 & $0,94^{* *}$ \\
\hline $\mathrm{K}: \mathrm{Mg}$ & 1,01 & 1,70 & 0,54 & 26,82 & $0,93^{* *}$ & 0,39 & 0,90 & 0,18 & 34,50 & $0,92^{* *}$ \\
\hline $\mathrm{K}:(\mathrm{Ca}+\mathrm{Mg})$ & 0,21 & 0,32 & 0,13 & 23,53 & $0,93^{* *}$ & 0,06 & 0,12 & 0,03 & 33,78 & $0,93^{* *}$ \\
\hline К/CTC & 0,15 & 0,22 & 0,10 & 20,01 & $0,94^{* *}$ & 0,05 & 0,08 & 0,02 & 28,02 & $0,93^{* *}$ \\
\hline Ca:Mg & 3,72 & 4,86 & 2,50 & 13,28 & $0,98^{\text {ns }}$ & 5,43 & 13,07 & 2,61 & 46,88 & $0,70^{* *}$ \\
\hline $\mathrm{Ca} / \mathrm{CTC}$ & 0,54 & 0,61 & 0,46 & 5,09 & $0,98^{\text {ns }}$ & 0,64 & 0,78 & 0,49 & 10,54 & $0,97^{\text {ns }}$ \\
\hline $\mathrm{Mg} / \mathrm{CTC}$ & 0,15 & 0,20 & 0,11 & 13,24 & $0,94^{* *}$ & 0,13 & 0,21 & 0,06 & 26,62 & $0,89^{* *}$ \\
\hline $\mathrm{Na}: \mathrm{Ca}$ & 0,14 & 0,23 & 0,08 & 23,89 & $0,95^{*}$ & 0,11 & 0,28 & 0,03 & 48,72 & $0,92^{* *}$ \\
\hline $\mathrm{Na}: \mathrm{Mg}$ & 0,52 & 0,89 & 0,28 & 27,62 & $0,96^{\mathrm{ns}}$ & 0,60 & 1,50 & 0,14 & 60,59 & $0,89^{* *}$ \\
\hline PST (\%) & 7,54 & 10,53 & 4,38 & 20,51 & $0,96^{\mathrm{ns}}$ & 7,03 & 14,75 & 2,26 & 42,35 & $0,95^{*}$ \\
\hline
\end{tabular}

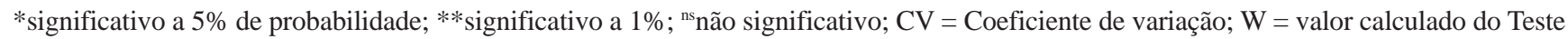
de Shapiro-Wilk; SST = teor de sólidos solúveis totais; PST = percentagem de sódio trocável (*significant at 5\% probability; **significant

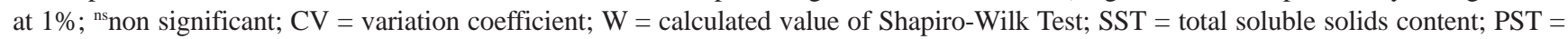
exchangeable sodium percentage).

valores citados por Kelling \& Peters (2004), indicam que o LVA apresentou alta saturação por $\mathrm{K}^{+}(15 \%)$, baixa saturação por $\mathrm{Ca}^{2+}(54 \%)$ e valores de $\mathrm{Mg}^{2+}$ acima dos adequados; no PVA, os valores de saturação por $\mathrm{K}^{+}$foram adequados (5\%), existindo locais com excesso (até $20 \%$ ), os de $\mathrm{Ca}^{2+}$ foram adequados $(64 \%)$, havendo locais com valores baixos, $\mathrm{e} \mathrm{o} \mathrm{Mg}^{2+}$ apresentou tanto valores adequados como elevados. Os dados originais dos dois solos, apresentados por Miranda (2004), indicam grande variabilidade dos teores de nutrientes, apesar das médias adequadas e valores de $\mathrm{pH}$ dentro da faixa ideal.

Considerando-se adequada para melão a relação Ca:Mg no solo de 3:1 (Silva, 2000), verificou-se excesso do $\mathrm{Ca}^{2+}$ em relação a $\mathrm{Mg}^{2+}$ no LVA, cuja média foi 3,72:1, mas variou entre 2,5:1 e 4,9:1 e, principalmente no PVA, onde Ca:Mg foi 5,43:1, com variação entre 2,6 e 13:1. Em geral, não são encontrados resultados significativos em ensaios com esta relação, como foi o caso da relação $2: 1$, mesmo tendo se mostrado mais favorável para matéria seca de plantas de feijoeiro (Oliveira \& Parra, 2003).

$\mathrm{O}$ excesso de $\mathrm{K}^{+}$em relação ao $\mathrm{Ca}^{2+}$, verificado no LVA, é indicado pela relação média de $027(1: 3,7)$, variando entre $0,41(1: 2,4)$ e $0,17(1: 5,9)$, enquanto é considerada adequada para melão a relação 1:9 $(0,11)$, segundo Silva (2000). Da mesma maneira, a média de 12,5 vezes mais $\mathrm{Ca}^{2+}(0,08)$, variando entre 7,1 $(0,14)$ e 33,3 vezes $(0,03)$, indica excesso de $\mathrm{Ca}^{2+}$ em relação a $\mathrm{K}^{+}$no PVA. Se considerada a relação K:Mg de 1:3 (0,33), preconizada por Silva (2000) como adequada, a relação média de 1:1, variando entre 1,85 vezes mais $\mathrm{Mg}^{2+}(0,54)$ e 1,7 vezes mais $\mathrm{K}^{+}$, indica excesso de $\mathrm{K}^{+}$em relação a $\mathrm{Mg}^{2+}$ no LVA. Em contrapartida, no PVA, a variação entre $1,1(0,90)$ e 14,2 vezes mais $\mathrm{Mg}^{2+}$ do que $\mathrm{K}^{+}(0,18)$ indica existirem locais com excesso de $\mathrm{Mg}^{2+}$.

Outra indicação dos teores muito elevados de $\mathrm{K}^{+}$no LVA é a relação $\mathrm{K}:(\mathrm{Ca}+\mathrm{Mg})$, cuja média foi 0,21 , com variação entre 0,13 e 0,32 , enquanto no PVA a média foi 0,06 com variação entre 0,03 e 0,12 . Os maiores valores desta relação indicam predominância de $\mathrm{K}^{+} \mathrm{em}$

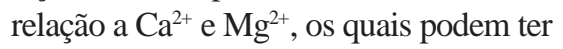
sua absorção reduzida, como foi observado em alfafa por Moreira et al. (2005).

Apesar de representar em média apenas 11 a $14 \%$ dos teores de $\mathrm{Ca}^{2+}$ nos dois solos, $\mathrm{o} \mathrm{Na}^{+}$pode representar risco em locais no PVA onde seus teores correspondiam a até $28 \%$ do $\mathrm{Ca}^{2+}$. Além disso, os teores de $\mathrm{Na}^{+}$representavam 52 a $60 \%$ dos de $\mathrm{Mg}^{2+}$, chegando a $150 \%$ em alguns locais do PVA. As PST médias de 7,54\% no LVA e 7,03\% no PVA não seriam prejudiciais para o melão, segundo Fipps (2003), que classifica como extremamente sensíveis culturas afetadas por PST entre 2 e 10 e sensíveis as afetadas por PST entre 10 e 20. No entanto, solos com PST maior do que $6 \%$ podem apresentar problemas de estabilidade estrutural (Van de Graaf \& Patterson, 2001).

A espessura de polpa do Goldex correlacionou-se positivamente $(\mathrm{P}<0,05)$ apenas com K:CTC (Tabela 2); o que não ocorreu significativamente no Orange Flesh $(\mathrm{P}>0,1)$. Isto indica a possibilidade de que a espessura de polpa tenha sido prejudicada pela maior disponibilidade de $\mathrm{Ca}^{2+}$ e favorecida pela maior disponibilidade de $\mathrm{K}^{+}$, o que está de acordo com Lester et al. (2005) que obtiveram maiores teores de açúcares, vitamina $\mathrm{C}$ e beta caroteno do Orange Flesh, além de melhor firmeza e coloração, devido à fertilização suplementar com potássio durante o desenvolvimento de frutos e maturação.

A firmeza de polpa do Goldex correlacionou-se positivamente com a relação $\mathrm{Mg}$ :CTC $(\mathrm{P}<0,01)$ e com $\mathrm{Ca}$ :CTC $(\mathrm{P}<0,1)$. Marschner (1995) destaca a importância do $\mathrm{Mg}^{2+}$ por ser o átomo central da molécula de clorofila; ser requerido ou promover fortemente várias enzimas e reações enzimáticas; re- 
Tabela 2. Correlações de Spearman entre variáveis de qualidade dos frutos de melão Goldex e Orange Flesh e relações catiônicas dos solos de cultivo (Spearman correlations between quality characteristics of Goldex and Orange Flesh melons and soil cationic ratios). Mossoró, UFERSA, 2002.

\begin{tabular}{|c|c|c|c|c|c|c|}
\hline \multirow{2}{*}{ Variáve } & \multicolumn{2}{|c|}{ Espessura } & \multicolumn{2}{|c|}{ Firmeza } & \multicolumn{2}{|c|}{ SST } \\
\hline & Goldex & O. Flesh & Goldex & O. Flesh & Goldex & O. Flesh \\
\hline $\mathrm{K}: \mathrm{Ca}$ & 0,19 ns & $0,16^{n s}$ & $-0,09 \mathrm{~ns}$ & $-0,22^{+}$ & $0,20^{+}$ & $0,14^{\mathrm{ns}}$ \\
\hline $\mathrm{K}: \mathrm{Mg}$ & 0,09 ns & 0,09 ns & $-0,16 \mathrm{~ns}$ & $-0,26$ * & $0,18^{\mathrm{ns}}$ & 0,09 ns \\
\hline $\mathrm{K}:(\mathrm{Ca}+\mathrm{Mg})$ & $0,18^{n s}$ & $0,14^{n s}$ & $-0,10 \mathrm{~ns}$ & $-0,24$ * & $0,20^{+}$ & 0,16 ns \\
\hline $\mathrm{K}: \mathrm{CTC}$ & 0,22 * & $0,21^{\mathrm{ns}}$ & $-0,03^{n s}$ & $-0,20 \mathrm{~ns}$ & $0,19^{+}$ & $0,14^{\mathrm{ns}}$ \\
\hline Ca:Mg & $-0,14 \mathrm{~ns}$ & $-0,21 \mathrm{~ns}$ & $-0,16 \mathrm{~ns}$ & $-0,11 \mathrm{~ns}$ & $0,01 \mathrm{~ns}$ & $-0,02 \mathrm{~ns}$ \\
\hline $\mathrm{Ca} / \mathrm{CTC}$ & $-0,09 \mathrm{~ns}$ & $-0,01^{\mathrm{ns}}$ & $0,19^{+}$ & 0,27 * & $-0,18^{n s}$ & $-0,13^{n s}$ \\
\hline $\mathrm{Mg} / \mathrm{CTC}$ & $0,11^{\mathrm{ns}}$ & 0,20 ns & 0,28 * * & $0,14^{\text {ns }}$ & $-0,09 \mathrm{~ns}$ & $0,01^{n s}$ \\
\hline $\mathrm{Na} \mathrm{Ca}$ & $0,13^{\mathrm{ns}}$ & $-0,01 \mathrm{~ns}$ & $-0,13^{n s}$ & $-0,25$ * & $0,19^{+}$ & $0,15^{n s}$ \\
\hline $\mathrm{Na}: \mathrm{Mg}$ & 0,04 ns & $-0,07$ ns & $-0,16 \mathrm{~ns}$ & $-0,20 \mathrm{~ns}$ & $0,19^{+}$ & 0,06 ns \\
\hline PST & 0,12 ns & $-0,01 \mathrm{~ns}$ & $-0,10 \mathrm{~ns}$ & $-0,20 \mathrm{~ns}$ & 0,16 ns & 0,12 ns \\
\hline
\end{tabular}

** significativo a $1 \%$ de probabilidade; *significativo a $5 \% \mathrm{e}^{+}$significativo a $10 \%$; SST $=$ teor de sólidos solúveis totais; PST = percentagem de sódio trocável $(* *$ significant at $1 \%$; *significant at $5 \%$ probability and +significant at $10 \%$; SST $=$ total soluble solids content; PST $=$ exchangeable sodium percentage).

Tabela 3. Estimativas dos parâmetros, somas de quadrados do tipo II parciais e coeficientes de determinação parciais das regressões múltiplas das variáveis de qualidade dos frutos de melão Goldex e Orange Flesh em função de relações catiônicas dos solos amostrados (estimative of parameters, partial type II sum of squares and partial determination coefficients of multiple regressions of quality characteristics of Goldex and Orange Flesh melons as a function of soil cationic ratios). Mossoró, UFERSA, 2002.

\begin{tabular}{|c|c|c|c|c|c|c|c|}
\hline \multirow{2}{*}{$\begin{array}{l}\text { Espessura } \\
\text { de polpa }\end{array}$} & \multicolumn{3}{|c|}{$\begin{array}{c}\text { Goldex - Latossolo } \\
\left(R^{2} 0,99\right)\end{array}$} & & \multicolumn{3}{|c|}{$\begin{array}{c}\text { Orange Flesh - Argissolo } \\
\left(R^{2} 0,99\right)\end{array}$} \\
\hline & EP & SQ II & $R^{2}(\%)$ & & EP & SQ II & $R^{2}(\%)$ \\
\hline $\mathrm{K}: \mathrm{Mg}$ & $-3,09$ & $0,49^{* *}$ & 0,04 & $\mathrm{~K} / \mathrm{Ca}$ & 10,95 & $3,55^{* *}$ & 0,60 \\
\hline Ca:Mg & 0,63 & $1,37^{* *}$ & 0,12 & $\mathrm{Ca} / \mathrm{CTC}$ & 4,80 & $39,35^{\star *}$ & 6,19 \\
\hline $\mathrm{Mg} / \mathrm{CTC}$ & 10,66 & $0,73^{* *}$ & 0,06 & - & - & - & - \\
\hline $\mathrm{Na}: \mathrm{Mg}$ & 2,81 & $0,22^{*}$ & 0,02 & - & - & - & - \\
\hline PST & $-0,21$ & $0,23^{*}$ & 0,02 & - & - & - & - \\
\hline $\mathrm{K}:(\mathrm{Ca}+\mathrm{Mg})$ & 15,74 & $0,60^{* *}$ & 0,05 & - & - & - & - \\
\hline \multirow{2}{*}{ Firmeza } & \multicolumn{3}{|c|}{$R^{2} 0,99$} & & \multicolumn{3}{|c|}{$R^{2} 0,99$} \\
\hline & EP & SQ II & $\mathbf{R}^{2}(\%)$ & & EP & SQ II & $\mathbf{R}^{2}(\%)$ \\
\hline $\mathrm{K}: \mathrm{Mg}$ & $-37,94$ & $71,84^{* *}$ & 0,06 & - & - & - & - \\
\hline $\mathrm{Ca} / \mathrm{CTC}$ & 33,14 & $46,68^{*}$ & 0,04 & $\mathrm{Ca} / \mathrm{CTC}$ & 78,54 & $12853,57^{* *}$ & 10,91 \\
\hline $\mathrm{Mg} / \mathrm{CTC}$ & 120,87 & $47,35^{*}$ & 0,04 & PST & $-3,91$ & $69,56^{*}$ & 0,06 \\
\hline $\mathrm{Na}: \mathrm{Ca}$ & $-278,87$ & $120,14^{* *}$ & 0,10 & $\mathrm{Na}: \mathrm{Ca}$ & 337,82 & $196,28^{* *}$ & 0,17 \\
\hline $\mathrm{Na}: \mathrm{Mg}$ & 75,05 & $128,40^{* *}$ & 0,11 & $\mathrm{Na}: \mathrm{Mg}$ & $-14,01$ & $45,59^{* *}$ & 0,04 \\
\hline $\mathrm{K}:(\mathrm{Ca}+\mathrm{Mg})$ & 199,15 & $82,93^{* *}$ & 0,07 & - & - & - & - \\
\hline \multirow{2}{*}{ SST } & \multicolumn{3}{|c|}{$R^{2} 0,99$} & & \multicolumn{3}{|c|}{$R^{2} 0,99$} \\
\hline & EP & SQ II & $\mathbf{R}^{2}(\%)$ & & EP & SQ II & $\mathbf{R}^{2}(\%)$ \\
\hline $\mathrm{K}: \mathrm{Mg}$ & 6,43 & $13,81^{* *}$ & 0,13 & $\mathrm{~K}: \mathrm{Mg}$ & 2,79 & $2,40^{*}$ & 0,07 \\
\hline KICTC & $-36,16$ & $6,32^{* *}$ & 0,06 & Ca:Mg & 0,85 & $27,90^{* *}$ & 0,80 \\
\hline $\mathrm{Ca} / \mathrm{CTC}$ & 8,73 & $7,91^{* *}$ & 0,07 & - & - & - & - \\
\hline $\mathrm{Mg} / \mathrm{CTC}$ & 42,00 & $11,43^{* *}$ & 0,11 & $\mathrm{Mg} / \mathrm{CTC}$ & 29,79 & $52,22^{* *}$ & 1,54 \\
\hline
\end{tabular}

$\mathrm{R}^{2}=$ coeficiente de determinação; $\mathrm{SST}=$ teor de sólidos solúveis totais; $\mathrm{EP}=$ estimativa do parâmetro; SQ II = soma dos quadrados do tipo II; PST = percentagem de sódio trocável; ** significativo a $1 \%$ de probabilidade; * significativo a $5 \%\left(\mathrm{R}^{2}=\right.$ coefficient of determination; $\mathrm{SST}=$ total soluble solids content; $\mathrm{EP}=$ the estimative of parameters; SQ II = type II sum of squares; PST = exchangeable sodium percentage; ${ }^{* *}$ significant at $1 \%$ probability; ${ }^{*}$ significant at $5 \%$ ). gular o $\mathrm{pH}$ celular e o equilíbrio cátion ânion; atuar em processos necessários à síntese de proteínas, formação de RNA, transferência de elétrons e síntese de ATP. Além disso, plantas deficientes em $\mathrm{Mg}^{2+}$ acumulam amido e açúcares nas folhas, com menor translocação para tecidos de armazenamento de frutos, sementes, raízes e tubérculos. No Orange Flesh a firmeza teve correlações negativas $(\mathrm{P}<0,05)$ com $\mathrm{K}: \mathrm{Mg}$ e $\mathrm{K}:(\mathrm{Ca}+\mathrm{Mg})$ e positiva com $\mathrm{Ca}$ :CTC, além de correlação negativa $(\mathrm{P}<0,1)$ com $\mathrm{K}: \mathrm{Ca}$. O Ca $\mathrm{Ca}^{2+}$ influencia a qualidade dos frutos de melão devido à sua função na estrutura das células, melhorando a textura e proporcionando frutos firmes e consistentes (Faria \& Fontes, 2003).

O teor de sólidos solúveis totais do Orange Flesh não se correlacionou com as relações catiônicas, apesar da correlação positiva com o teor de $\mathrm{Mg}^{2+}$ observada por Miranda (2004). No Goldex as correlações positivas $(\mathrm{P}<0,1)$ com as relações $\mathrm{K}: \mathrm{Ca}, \mathrm{K}: \mathrm{CTC}, \mathrm{K}:(\mathrm{Ca}+\mathrm{Mg})$, $\mathrm{Na}: \mathrm{Ca}$ e $\mathrm{Na}: \mathrm{Mg}$, podem indicar a ocorrência das maiores SST nos locais com maior disponibilidade de $\mathrm{K}^{+}$e onde seus teores tendiam a se equilibrar com os de $\mathrm{Ca}^{2+}$. Além de SST, o K ${ }^{+}$trocável no solo atua sobre tamanho, espessura, coloração da casca e acidez de frutos, e promove o aumento nos teores de carboidratos, óleos, gorduras e proteínas nos produtos agrícolas (Malavolta et al., 1997; Faria \& Fontes, 2003). A influência positiva das relações envolvendo $\mathrm{Na}^{+}$pode ser devida à atenuação da predominância no solo dos cátions

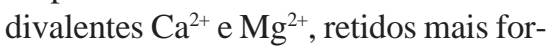
temente do que os monovalentes (Malavolta et al., 1997).

As regressões revelam a baixa contribuição das relações catiônicas para a variação das características de qualidade do melão (Tabela 3). A contribuição para espessura de polpa do Goldex das relações Ca:Mg $(0,12 \%)$ e Ca:CTC no Orange Flesh $(6,19 \%)$, cujas correlações foram negativas, levanta a possibilidade do excesso de $\mathrm{Ca}^{2+}$ ser causa importante do desequilíbrio entre os teores de cátions trocáveis do solo.

A maior contribuição para a firmeza de polpa do Goldex, das relações $\mathrm{Na}: \mathrm{Ca}$ e $\mathrm{Na}: \mathrm{Mg}$, esta última também no 
Orange Flesh, as quais têm correlação negativa, indica que o excesso de $\mathrm{Na}^{+}$

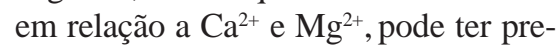
judicado a absorção destes cátions. A contribuição destacada de Ca:CTC $(10,9 \%)$ no Orange Flesh, com correlação positiva, reforça a influência de teores elevados de $\mathrm{Ca}^{2+}$ no aumento da firmeza de polpa, tão importante que Marschner (1995) cita que a elevação no teor de $\mathrm{Ca}^{2+}$ nos frutos causa aumento na firmeza e atraso no amadurecimento, enquanto que, em tecidos de frutos de plantas com deficiência severa ocorre desintegração da estrutura das membranas e vazamento de solutos de baixo peso molecular.

O teor de SST do Goldex teve as maiores contribuições de $\mathrm{K}: \mathrm{Mg}$ e $\mathrm{Mg}: \mathrm{CTC}$, este último foi o de maior contribuição no Orange Flesh, que também teve contribuição de $\mathrm{Ca}: \mathrm{Mg}$. Isto indica a importância tanto do $\mathrm{Mg}^{2+}$, como já discutido, como do $\mathrm{K}^{+}$, que influencia o teor de açúcares nos frutos de melão, atuando no carregamento e descarregamento da sacarose do floema para os frutos, mas cuja absorção durante o desenvolvimento de frutos e maturação pode ser inadequada se houver competição pela absorção com $\mathrm{Ca}^{2+}$ e $\mathrm{Mg}^{2+}$ (Lester et al. 2005).

As variáveis de qualidade dos frutos de melão apresentaram poucas correlações com as relações catiônicas do solo. No Goldex, as melhores correlações foram de K:CTC com espessura de polpa; Ca:CTC e Mg:CTC com firmeza de polpa e K:CTC, K:Ca, $\mathrm{K}:(\mathrm{Ca}+\mathrm{Mg}), \mathrm{Na}: \mathrm{Ca}$ e Na:Mg com SST, todas positivas. No Orange Flesh, observou-se correlações apenas de firmeza de polpa com Ca:CTC (positiva), $\mathrm{K}: \mathrm{Mg}, \mathrm{K}: \mathrm{Ca}, \mathrm{K}:(\mathrm{Ca}+\mathrm{Mg})$ e Na:Ca (negativas). No Orange Flesh, através da contribuição para as regressões, identificou-se como mais importantes as re- lações catiônicas Ca:CTC para espessura $(6,2 \%)$ e firmeza de polpa $(10,9 \%)$, e Mg:CTC para SST (1,5\%).

\section{REFERÊNCIAS}

BERGEZ JE; NOLLEAU S. 2003. Maize grain yield variability between irrigation stands: a theoretical study. Agricultural Water Management 60: 43-57.

CRISÓSTOMO LA; SANTOS AA; RAIJ B; FARIA CMB; SILVADJ; FERNANDES FAM; SANTOS FJS; CRISÓSTOMO JR; FREITAS JAD; HOLANDA JS; CARDOSO JW; COSTA ND. 2002. Adubação, irrigação, híbridos $e$ práticas culturais para o meloeiro no Nordeste. Fortaleza: Embrapa Agroindústria Tropical. 21p. (Circular Técnica 14)

EMBRAPA. 1997. Manual de métodos de análises de solos. Rio de Janeiro: Embrapa. 212p. (Documentos, 1)

FARIA CMB; FONTES RR. 2003. Nutrição e adubação. In: SILVA, H.R.; COSTA, N.D. Melão: Produção Aspectos técnicos. Brasília: Embrapa Hortaliças, Embrapa Semi-Árido. p.40-50.

FILGUEIRAS HAC; MENEZES JB; ALVES RE; COSTA FV; PEREIRA LSE; GOMES JÚNIOR J. 2000. Colheita e manuseio póscolheita. In: ALVES RE. (ed.). Melão: PósColheita. Brasília: EMBRAPA. p.23-41.

FIPPS G. 2003. Irrigation water quality standards and salinity management. College StationTexas: Texas Cooperative Extension; Texas A \& M University. 20p.

FRANÇA GE; OLIVEIRA AI; MANTOVANI EC; ANDRADE CLT; MARRIEL IE. 2000. Análise preliminar de mapas de variabilidade espacial da fertilidade do solo. In: BORÉM A; DEL GIUDICE MP; QUEIROZ DM; MANTOVANI EC; FERREIRA LR; VALLE FXR; GOMIDE RL. Agricultura de precisão. Viçosa: UFV. p.321-338.

GONÇALVES ACA; FOLEGATTI MV; VIEIRA SR. 1999. Padrões de amostragem e intensidade de krigagem na caracterização do armazenamento de água no solo, em área irrigada por pivô central. Revista Brasileira da Ciência do Solo 23: 485-495.

KELLING KA; PETERS JB. 2004. The advisability of using cation balance as a basis for fertilizer recommendations. 2004 Wisconsin Fertlizer, Aglime, \& Pest Management Conference Proceedings. Disponível em http:// www.soils.wisc.edu/ extension/ FAPM/ 2004proceedings/Kelling1.pdf. Acessado em 21 de novembro de 2006.
LESTER GE; JIFON JL; ROGERS G. 2005. Supplemental foliar potassium application to muskmelon (Cucumis Melo L.) during fruit growth improves quality and content of human wellness components. Journal of American Society of Horticultural Science 130: 649-653.

MALAVOLTA E; VITTI GC; OLIVEIRA SA. 1997. Avaliação do estado nutricional das plantas: princípios e aplicações. Piracicaba: POTAFOS. 319p.

MARSCHNER H. 1995. Mineral nutrition of higher plants. 2. ed. New York: Academic Press. 889 p.

MIRANDA NO; OLIVEIRA TS; LEVIEN SLA; SOUZA ER. 2005. Variabilidade espacial da qualidade de frutos de melão em áreas fertirrigadas. Horticultura Brasileira 23: 244-251.

MIRANDA NO. 2004. Variabilidade espacial de componentes de produção na cultura do meloeiro fertirrigado. Fortaleza: UFC. 135p. (Tese doutorado)

MOREIRAA; CARVALHO JG; EVANGELISTA AR. 2005. Relação cálcio e magnésio na fertilidade de um latossolo vermelho escuro distrófico cultivado com alfafa. Ciência Agrotecnologia 29: 786-794.

OLIVEIRA EL; PARRA MS. 2003. Resposta do feijoeiro a relações variáveis entre cálcio e magnésio na capacidade de troca de cátions de latossolos. Revista Brasileira da Ciência do Solo 27: 859-866.

SCHLINDWEIN JA; ANGHINONI I. 2000. Variabilidade horizontal de atributos de fertilidade e amostragem do solo no sistema plantio direto. Revista Brasileira da Ciência do Solo 24: 85-91.

SILVA HR; COSTA ND. 2003. Introdução. In: SILVA, H.R.; COSTA, N.D. Melão: Produção aspectos técnicos. Brasília: Embrapa Hortaliças; Embrapa Semi-Árido. p.12-14.

SILVA JR. 2000. Efeito do equilíbrio catiônico do solo na produção e qualidade de frutos de melão (Cucumis melo L.). Fortaleza: UFC. 57 p. (Tese mestrado)

UPADHYAYA SK; ROSA UA; EHSANI M; KOLLER M; JOSIAH M; SHIKANAI T. 1999. Precision farming in a tomato production system. St. Joseph: ASAE. 23p. (ASAE paper $\mathrm{N}^{\circ}$. 991147).

VAN DE GRAAF R; PATTERSON RA. 2001. Explaining the mysteries of salinity, sodicity, SAR and ESP in on-site practice. In: PATTERSON RA; JONES MJ. (eds.) Proceedings of on-site '01 Conference: advancing on-site wastewater systems. Armidale: Lanfax Laboratories. p.361-368. 